\title{
El modelo de juego en el fútbol. De la concepción teórica al diseño práctico Game models in soccer. From theoretical conception to practical design \\ *Alberto Martín-Barrero, **Francisco Ignacio Martínez-Cabrera \\ *Centro Universitario San Isidoro (España), **Universidad Pablo de Olavide (España)
}

\begin{abstract}
Resumen. En los últimos años hemos visto una tendencia hacia un cambio en los conceptos y metodologías de entrenamiento de fútbol. El paradigma tradicional ha dado paso a nuevas propuestas que entienden el juego desde un plano holístico. Esta ruptura ha supuesto la aparición de nuevas formas de concebir el entrenamiento, emergiendo métodos de entrenamiento que se acercan a la especificidad del deporte. A día de hoy, contamos con diversos métodos de trabajo, entre los cuales podríamos citar el microciclo estructurado y la periodización táctica como lo que mayor interés y repercusión han suscitado debido a su utilización por entrenadores de éxito. En este sentido, la periodización táctica se fundamenta en la concepción del modelo de juego como elemento fundamental de donde parten los diferentes patrones de juego que deben ser reproducidos posteriormente en el entrenamiento. Debido a la falta de propuesta prácticas que manifiesten pautas claras para el desarrollo de un modelo de juego, en este trabajo trataremos de llevar a cabo un diseño propio a partir de i) un análisis conceptual sobre el modelo de juego, ii) identificación de los elementos que este debe de contener y iii) desarrollo práctico de cada uno de los elementos que sirva como hoja de ruta para aquellos profesionales que quieran profundizar en esta forma de concebir el entrenamiento en fútbol.
\end{abstract}

Palabras clave: fútbol, modelo de juego, periodización táctica

\begin{abstract}
In recent years, we have identified a trend towards a change in the concepts and methodologies of soccer training. The traditional paradigm has given way to new proposals that understand the game from a holistic perspective. This break has led to the emergence of new ways of conceiving training. These training methods are close to each sport's specificity. One of the methods that has raised more interest, due to its repercussions in professional soccer thanks to successful coaches, is the Tactical Periodization. This idea is based on the conception of game model as a fundamental element from which different game patterns grow, each of them needing to be reproduced later in the training. On account of a lack of practical proposals showing clear guidelines for the development of a game model, we try to present a personal design starting from i) a conceptual analysis of the game model, ii) the identification of the elements that this model needs to show, and iii) a practical development of each element, which serves as route map for those professionals who wish to delve into this training approach in soccer.
\end{abstract}

Key Words: soccer, game model, tactical periodization.

\section{Introducción}

En las últimas décadas, el fútbol se ha convertido en una materia de estudio desde un punto de vista multidisciplinar. Podemos encontrar numerosos trabajos e investigaciones relacionadas con el análisis del rendimiento desde el plano de la condición física, desde la perspectiva psicológica y ahora, en los últimos tiempos, son numerosos los autores e investigadores que han pasado a analizar el rendimiento en fútbol desde una perspectiva táctica (Drust, Atkinson y Reilly, 2007; Reilly, 2005).

Las nuevas teorías y paradigmas surgidos en torno a la ciencia del deporte invitan a que el fútbol se estudie y analice desde un prisma diferente. Autoras como Balagué y Torrents (2011) sugieren que el deportista es un sistema complejo que depende de una red de interrelaciones que actúan como un todo y sobre las cuales emergen sus diferentes comportamientos a través de las diferentes sinergias de las mismas. Este cambio de paradigma está extendiéndose a diferentes investigadores, ayudando a proporcionar una visión más amplia del análisis del rendimiento y del juego, urgiendo la necesidad de asumir una nueva perspectiva científica, el paradigma de la complejidad, el cual encara la realidad con una visión unificadora, alejando la noción cartesiana (Silva, Oliveira y Kautzner, 2009). A su vez, este hecho ha llevado al fútbol a evolucionar desde una perspectiva sistémica, donde debe ser analizado como un todo indepen-

Fecha recepción: 12-02-19. Fecha de aceptación: 15-05-19

Alberto Martín Barrero

amarbar10@gmail.com dientemente de sus elementos tácticos, técnicos, fisiológicos y psicológicos (Olivera, 2004). Por lo tanto, el fútbol ha pasado de analizarse desde una perspectiva y metodología reduccionista a una metodología que comprende la globalidad de los factores y momentos que componen el fútbol (Tamarit, 2010), y, por tanto, entendida desde un prisma que intente plasmar en el entrenamiento las situaciones reales de juego (Serra et al., 2011).

Desde este enfoque nacen nuevas ideas y formas de entender la concepción del entrenamiento del fútbol. Tal como indican Garganta y Pinto (1998), un jugador de fútbol es aquel que es capaz de seleccionar la acción más apropiada para responder a diferentes situaciones de juego, y estas acciones siempre corresponden a una dimensión táctica. En base a este concepto se fundamentan algunas de las corrientes metodológicas más empleadas actualmente en el fúbol, como son el microciclo estructurado y la periodización táctica (PT). Esta última (PT) se basa en organizar y construir el proceso de entrenamiento con el fin de llevarlo a cabo en la competición (Frade, 1985). Es entonces cuando surge la concepción de juego, definida desde el «cómo» un entrenador quiere que su equipo juegue, planteando diferentes patrones de juego que posteriormente deben ser reproducidos en el entrenamiento basados en un principio fundamental como es la imprevisibilidad (Delgado y Méndez-Villanueva, 2012). Dentro de esta nueva perspectiva, la figura del entrenador aparece como aquel que debe «construir» y configurar los diferentes patrones de juego que permitan desarrollar ese «fútbol». Siguiendo esta premisa, Tamarit (2010) define esta idea como «cultura táctica» del entrenador, siendo permanentemente consciente del diseño y proceso tanto del juego 
como del entrenamiento. Además, habría que añadir que este proceso es de larga duración, matiz el cual nos hace entender que este proceso debe ser bien definido desde el principio pero que, a su vez, tiene un carácter abierto y flexible que puede ser modificado en el tiempo.

En las siguientes páginas se pretende analizar la concepción teórica del modelo de juego (MJ) como parte elemental de esta forma de entender el proceso de entrenamiento en el fútbol y a la misma vez lanzar una propuesta sobre qué elementos pueden componer y se deben tener en cuenta para poder diseñar un MJ.

\section{Concepción teórica sobre el modelo de juego en el} fútbol

Son numerosos los técnicos, entrenadores e investigadores que tratan de conceptualizar y expresar el significado sobre el MJ (Oliveira, 2004; Portolés, 2007, citado en Tamarit, 2010; Tamarit, 2010; Cano, 2012; Delgado y MéndezVillanueva, 2012; Mourinho, 2014; Guindos, 2015; Garganta, 2016; Martín-Barrero, 2016). Nos obstante, en el contexto del fútbol aún hay cierta confusión en relación a este concepto. Aunque esta idea lleva desarrollándose desde hace tiempo, el MJ en fútbol es normalmente mal entendido. Muchos hablan de él como el sistema o esquema de juego empleado (Tamarit, 2010). De hecho son varias las investigaciones que proponen el sistema de juego (SJ) como variable o elemento principal en sus trabajos (Vales-Vázquez, 1998; Castelo, 1999; López, 2004; Gurutz, 2012 y Pascual, 2012). Para entender la diferencia entre un aspecto y otro, empezaremos a definir el concepto de SJ como el posicionamiento de los jugadores en el campo Delgado y Méndez-Villanueva (2012) o, para ser más conciso, como la distribución inicial de los jugadores en el terreno de juego, la cual permite la distribución de los jugadores en posiciones, facilitando así la organización posicional del equipo (Martín-Barrero, 2018).

En relación al MJ, Oliveira (2004) lo entiende como una idea/conjetura de juego constituida por principios, sub-principios, sub-principios de los sub-principios, etc., representativos de los diferentes momentos / fases de juego, que se articulan entre sí, manifestando una organización funcional propia, es decir, una identidad. Este concepto de identidad también lo defiende Portolés (2007), afirmando que el MJ es algo que identifica a un equipo determinado. Por su parte, Mourinho (2014) reafirma esta característica de identificación, definiéndolo como los principios que establezco en mi equipo, los principios prioritarios que nos otorga un cierto ADN (una identidad).

La organización de los elementos y componentes que conforman el juego, junto a la longevidad o regularidad de la misma, es una propiedad a resaltar en la definición de MJ. Para Amieiro (2005) la identidad de un equipo no es más que la afirmación regular de la organización que conforman. En este sentido, Tamarit (2010) indica que se trata de la organización que presenta un equipo en cada momento del juego y lo hace de forma regular. En relación a la prolongación en el tiempo, Frade (1985) (padre de la PT) indicó que el MJ debe de estar siempre en entredicho, manteniéndose el futuro como elemento causal del comportamiento. Carvalhal (2001) le da importancia a construir el MJ siempre como el futuro, como algo a alcanzar, a donde se pretende llegar.

Otra característica importante sobre el MJ que encontramos analizando la bibliografía es la importancia del jugador y sus interacciones dentro de este modelo u organización. Según Frade (2004) el juego tiene que nacer primero de la mente de los jugadores, resaltando y mejorando sus características. Para Cano (2012) el MJ es la organización de las tendencias conceptuales que se originan con la interacción de las capacidades naturales de quienes juegan. En línea con esta argumentación, Portolés (2007, citado en Tamarit, 2010) reafirma esta característica aportando que el MJ es la forma en la que esos jugadores se relacionan entre si y como expresan su forma de ver el fútbol. Por tanto, será esencial el análisis previo de las características y capacidades de sus jugadores con el objetivo de poder adaptar sus virtudes al conjunto del equipo al mismo tiempo que enmascaremos las debilidades individuales.

Aunque bien es cierto que el jugador es o debe ser el principal protagonista de un MJ, este debe ser diseñado, guiado y construido por parte del entrenador, el cual juega un rol crucial en este escenario. Por su parte, Freitas (2004) cita que el entrenador debe llevar y presentar a los jugadores una imagen del MJ en sus cabezas, constatando que el entrenador debe tener un rol muy importante en el diseño del entrenamiento y en la configuración de las tareas del mismo. Además de esta función, el entrenador debe permitir que el MJ desarrolle la capacidad de aprender a aprender, es decir, que lleve al jugador a autogestionar su proceso de entrenamiento y aprendizaje dentro del mismo (Martín-Barrero, 2018). Además de la figura del entrenador, dentro del cuerpo técnico de un equipo, los diferentes miembros del mismo, incluyendo una nueva perspectiva del preparador físico actual deben estar en la misma sintonía y hacer un trabajo multidisciplinar en el cual el punto de partida seas los principios tácticos (Guindos, 2015).

Hay otros autores que insisten en la importancia del MJ como hoja de ruta o guía. En este sentido, Carvalhal (2001) refuerza esta idea indicando que son las coordenadas para poder trabajar y llegar al máximo nivel de juego. Por su parte, Garganta (2016) indica que el MJ funciona como mapa que guía la ruta a profundizar. Dentro de esta forma de trabajar y organización del juego, es importante conocer la estructura que conforma este mapa o ruta, basada tal y como indica Tamarit (2010) en principios y subprincipios de juego, que son los comportamientos y la articulación de los mismos y que van a permitir tener una identidad de juego. Para Oliveira (2004) estos principios y subprincipios son aquellos comportamientos que el entrenador crea y quiere que asuma el equipo colectivamente y los jugadores individualmente. La capacidad de interpretación y la creatividad por parte de los verdaderos protagonistas, los jugadores, es un elemento característico que varios autores resaltan (Tamarit, 2010; Cano, 2012; Delgado y Méndez-Villanueva, 2012; MartínBarrero, 2018). Como uno de los últimos elementos que caracteriza al MJ encontramos la flexibilidad del mismo, ya que este debe permitir adaptarse a diferentes contextos y ser abierto según los jugadores y circunstancias de juego (Frade, 1985; Castelo, 1994; Tamarit, 2010, Cano, 2012 y Martín-Barrero, 2018).

La contextualización del MJ es un aspecto a señalar, ya 
que dependiendo de club o entidad deportiva y su respectiva historia, masa social, trayectoria deportiva, tipo o tipos de competiciones en las que suelen participar, el MJ cobrará mayor o menos relevancia y este, a su vez, debe ser variable dependiendo del contexto donde se introduzca (Tamarit, 2010) (Figura 1). Las condiciones culturales y de entrenamiento también deben ser tenidas en cuenta para implementar un MJ (Delgado y Méndez-Villanueva, 2012). Por lo tanto, el MJ está compuesto por diferentes características o elementos que le dan un carácter propio a este concepto, diferenciándolo claramente de la idea de SJ o, incluso, del estilo de juego. En la tabla 1 se pretende establecer un resumen de las diferentes características que definen al MJ como concepto, a la misma vez que se relacionan estas características con los autores y expertos que mencionan las mismas.

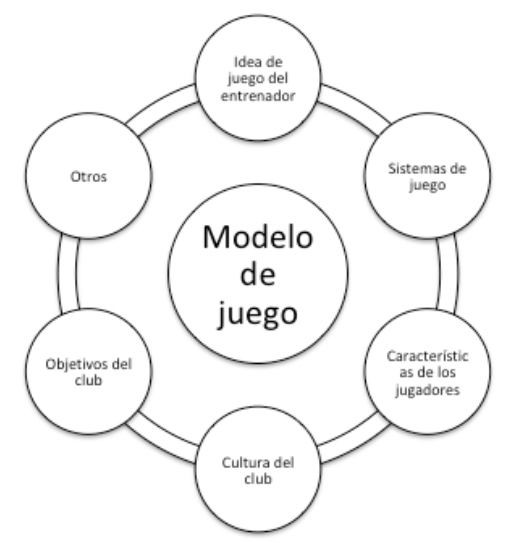

Figura 1. Factores que condiciona el MJ. Adaptado y modificado de Tamarit (2010)

\begin{tabular}{|c|c|c|}
\hline $\begin{array}{l}\text { Características del } \\
\text { MJ en fútbol }\end{array}$ & Aspectos a tener en cuenta & Autores \\
\hline Organización & Momentos/fases del del juego. & Amieiro, 2005; Tamarit 2010 \\
\hline Identidad & $\begin{array}{l}\text { Estilo de juego. } \\
\text { ADN. }\end{array}$ & $\begin{array}{l}\text { Oliveira, 2004; Portolés, 2007; } \\
\text { Mourinho, 2014 }\end{array}$ \\
\hline $\begin{array}{l}\text { Interacción de } \\
\text { jugadores }\end{array}$ & $\begin{array}{l}\text { Potenciador de las cualidades de los } \\
\text { jugadores y del equipo. }\end{array}$ & $\begin{array}{l}\text { Frade, 2004; Portolés, 2007; Cano, } \\
2012\end{array}$ \\
\hline $\begin{array}{c}\text { Perdurabilidad en el } \\
\text { tiempo }\end{array}$ & $\begin{array}{l}\text { Consecuencia a alcanzar. } \\
\text { Camino a construir. }\end{array}$ & Frade, 1985; Carvalhal, 2001 \\
\hline Guía & $\begin{array}{l}\text { Principios y subprincipios } \\
\text { (comportamientos). }\end{array}$ & $\begin{array}{l}\text { Carvalhal 2001; Oliveira 2004; } \\
\text { Tamarit 2010; Garganta, 2016 }\end{array}$ \\
\hline Creatividad & $\begin{array}{l}\text { Interpretación. } \\
\text { Mecanismo no mecánico. }\end{array}$ & $\begin{array}{l}\text { Tamarit, 2010, Cano, 2012; } \\
\text { Delgado y Méndez-Villanueva, } \\
2012 \text { y Martín-Barrero, } 2018\end{array}$ \\
\hline Flexibilidad & $\begin{array}{l}\text { Abierto y con capacidad de } \\
\text { adaptación a contextos. }\end{array}$ & $\begin{array}{l}\text { Frade, 1985; Castelo, 1994; } \\
\text { Tamarit, 2010, Cano, } 2012 \text { y } \\
\text { Martín-Barrero, } 2018\end{array}$ \\
\hline Contextualización & $\begin{array}{l}\text { A los clubes (historia, masa social). } \\
\text { Cultura. } \\
\text { Competición. }\end{array}$ & $\begin{array}{l}\text { Tamarit, 2010; Delgado y Méndez- } \\
\text { Villanueva, } 2012\end{array}$ \\
\hline Rol del entrenador & $\begin{array}{l}\text { Diseñador de los patrones de juego } \\
\text { El preparador físico como parte que } \\
\text { tiene que entender el MJ }\end{array}$ & $\begin{array}{l}\text { Guindos, 2015; Martín-Barrero, } \\
2018\end{array}$ \\
\hline
\end{tabular}

Este análisis sobre la conceptualización del MJ es tremendamente beneficioso, ya que nos permite conocer gran parte de los elementos que deben componer el diseño de un MJ. En las próximas páginas ese será nuestro objetivo establecer una guía que permita orientar y dirigir a los profesionales del fútbol en los diferentes componentes y elementos que deben tener en cuenta durante la elaboración de un MJ.

\section{Organización de los contenidos para el diseño de un modelo de juego. Elaboración de la hoja de ruta.}

Una vez analizadas las diferentes características que influyen en el desarrollo de un MJ, partiremos de esta base para poder llevar a cabo una hoja de ruta sobre el diseño del mismo. A continuación se desarrollarán los diferentes ele- mentos a tener en cuenta, el concepto de cada uno de ellos y los principales aspectos sobre los que reflexionar y estudiar para poder así estructurar un diseño que sirva de guía y orientación.

La estructura propuesta está basada en dos pilares fundamentales (Figura 2):

$\varnothing$ Los elementos formales (que proporcionan al diseño la forma sobre la cual se va a desarrollar el MJ y contiene todos aquellos elementos que permiten organizar y definir los rasgos más básicos del mismo).

$\varnothing$ Los elementos funcionales (dotan de actividad propia al MJ a través de los elementos formales, es el contenido profundo sobre los que se identifican y desarrollan los diferentes comportamientos). La articulación de estos dos elementos conforma la estructura completa sobre la que se diseña el MJ.

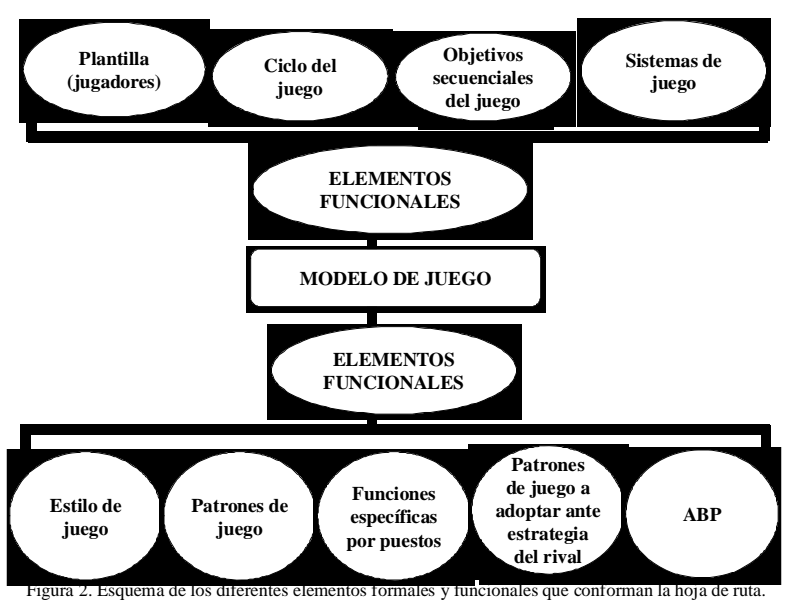

Ø Plantilla (jugadores):

Una de las características que resaltaban los diferentes autores analizados en el punto anterior de este trabajo es la importancia de los jugadores que componen el MJ. En esta propuesta, conocer y analizar a cada uno de ellos-as en relación a sus puntos fuertes, débiles y características más importantes (en diferentes parámetros de rendimiento: técnicotáctico, fisiológico, condicional, experiencia, etc.) es considerado el primer elemento que debe dar sentido a los siguientes contenidos. Además, en la actualidad, podemos obtener datos de rendimiento tanto técnico-táctico como físico que nos ayude a darle un mayor valor a este contenido mediante herramientas informáticas como WyScout, Mediacoah o InStat.

\section{$\varnothing$ Ciclo de juego:}

El juego se puede considerar como un ciclo compuesto por fases dinámicas y momentos de juego, y fases estáticas de juego [acciones a balón parado (ABP) o con balón en fuera de juego] (Castellano, 2000, 2008; Castellano, Perea y Álvarez, 2009), configurando el ciclo del juego, el cual es uno de los elementos que dotan de sentido organizativo al MJ y los podríamos definir como las diferentes fases y momentos por los que pasa el juego, estando determinadas por la dualidad que se establecen según quién es el poseedor del balón y el momento en el que lo ostenta. Siguiendo a Cano (2012), aunque el juego se puede dividir para ser analizado, este siempre debe ser visto y transmitido desde una visión 
holística. Las fases del juego son dos: ataque y defensa. Para los momentos del juego seguiremos la propuesta de Cano (2009) basados en 4 momentos: ataque organizado, contraataque, defensa organizada, organización defensiva. Basándonos en esta segunda propuesta podemos definir cada uno de los momentos del juego:

o Ataque organizado: momento ofensivo del juego en el cual el equipo rival se encuentra defensivamente organizado y por lo tanto no hay posibilidades de sorprenderle rápidamente.

o Contraataque: momento ofensivo en el cual puedo sorprender al equipo rival debido a que el mismo se encuentra desestructurado defensivamente y, por lo tanto, expuesto en ataque.

o Organización defensiva: momento defensivo en el cual el equipo se encuentra expuesto debido a la realización de un ataque.

o Defensa organizada: momento defensivo del juego en el cual el equipo rival no puede sorprendernos momentáneamente debido a que nuestro equipo está estructurado.

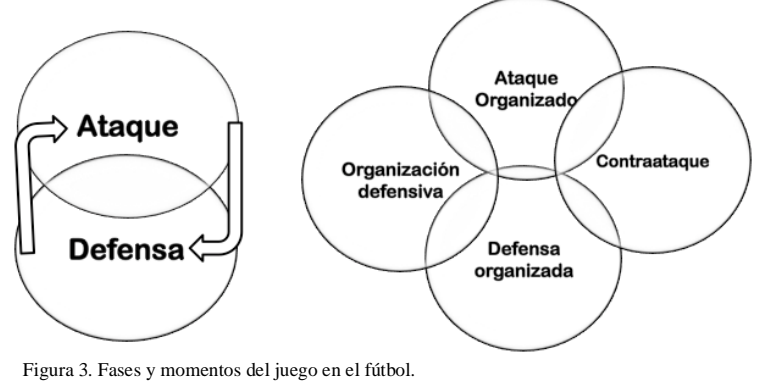

Ø Objetivos secuenciales del juego:

Podríamos considerar los objetivos secuenciales del juego (a partir de ahora OSJ) como la sucesión de finalidades para conseguir el objetivo fundamental. Autores como Marcos (2011) o Martín-Barrero (2016) utilizan este elemento como parte de sus trabajos en relación al análisis de la competición y al estudio del MJ. Los OSJ pueden ayudar a establecer un patrón que ayude a establecer los principios de cada uno de los momentos del juego. De tal forma que, para analizar y diseñar un MJ, cada momento del juego tendrán sus OSJ correspondientes según la fase de ataque o defensa, estableciéndose los siguientes criterios:

Ataque:

o Construir situaciones de ataque: son todas aquellas situaciones de saque de portero o de participación del mismo con la primera línea de jugadores atacantes con la intención de romper la $1^{\mathrm{a}}$ línea de defensa contraria.

o Construir situaciones de finalización: todos aquellos enlaces que se desarrollan en zonas avanzadas con la finalidad de conseguir desequilibrios que nos acerquen al área.

o Finalizar en situaciones favorables: aprovechar los desequilibrios cercanos al área y obtener las mejores situaciones para conseguir gol.

\section{Defensa:}

o Evitar la construcción de situaciones de ataque: son todas aquellas situaciones de saque de portero (rival) o participación del mismo con la primera línea ofensiva rival con la intención de que esta no rebase nuestra primera línea de presión o evite llevar el balón a zonas más avanzadas con dificultad.

o Evitar la construcción de situaciones de finalización: evitar todos aquellos enlaces o conexiones que el rival desarrolla en zonas avanzadas con el fin de conseguir desequilibrios que se acerquen a nuestra propia área.

o Evitar la finalización en situaciones favorables: evitar la penetración rival dentro del área y/o dificultar la finalización de cualquier acción.

\section{Ø Sistema de juego:}

El SJ desempeña un rol organizacional dentro de los diferentes elementos del MJ. Teodorescu (1984) hace relación al concepto de SJ con el de organización y estructura de un equipo. Esta estructura determina la posición y demarcación (portero, defensas, mediocentros y delanteros) de los jugadores en el campo. Además todo equipo establece un orden entre sus jugadores y sus diferentes líneas (Mercé, 2004) y estas líneas se expresan en cifras, es decir, un SJ 1-43-3 tendría 4 líneas de interrelación con un portero (primera línea), cuatro defensa (segunda línea), tres mediocentros (tercera línea) y tres delanteros (cuarta línea). En su relación directa como elemento en el MJ hay que resaltar que puede utilizarse más de un SJ, no siendo habitual encontrar más de 2-3 variantes.

\section{$\varnothing$ Estilo de juego:}

Comúnmente el estilo de juego es un término utilizado por entrenadores, científicos del deporte y analistas del rendimiento para referirse a la forma de jugar de un equipo o a los patrones más comunes utilizados por el mismo, pero, tal y como indica Hewitt, Greenham y Norton (2017), a pesar de esta definición tan generalizada, no existe una definición para estilo de juego. Fernández-Navarro, Fradua, Zubillaga, Ford y McRobert (2016) consideran el estilo de juego como los comportamientos generales de un equipo para conseguir los diferentes objetivos ofensivos y defensivos durante el juego. Siguiendo el trabajo de este autor podemos identificar estilos de juego ofensivos y defensivos. Los estilos de juego defensivos definidos por algunos autores son presión alta y presión baja (individual, zonal o mixta) (Bangsbo y Peitersen, 2000; Wright, Atkins, Polman, Jones y Sargeson, 2011), mientras que los estilos ofensivos identificados han sido juego directo, posesión y contraataque (Bate, 1988; Garganta, Maia y Basto, 1997; Tenga y Larsen, 2003; Hughes y Franks, 2005; Ruiz-Ruiz, Fradua, Fernández-García y Zubillaga, 2013; Fernández-Navarro y col., 2016).

\section{$\varnothing$ Patrones de juego:}

Podemos definir los patrones de juego como aquellos comportamientos que se llevan a cabo para conseguir unos determinados objetivos. Otros autores, sobre todos muy relacionados con la PT, relacionan este concepto de comportamientos o acciones de juego llevadas a cabo por los jugadores como principios, subprincipios y subprincipios de los subprincipios, en relación a la categoría que ocupan en el grado de concreción del comportamiento (Mourinho, J., en Fernandes, 2003; Oliveira, 2004; Tamarit, 2010; Delgado y Méndez-Villanueva, 2012). Nuestra propuesta es que los patrones de juego se engloben en una estructura mayor de- 
finida por 4 aspectos, de los cuales en el punto siguiente del trabajo se podrán ver diferentes ejemplos prácticos:

- Objetivo específico: planteados según intencionalidades ofensivas y defensivas.

Procedimientos de actuación: los comportamientos o acciones para conseguir el objetivo específico. Los medios técnico-tácticos son la base de la que parten los diferentes comportamientos y acciones tanto individuales, colectivas y de equipo, sin olvidar los aspectos actitudinales.

- Espacios: relacionados con las subdivisiones de zonas, tal y como otros autores e investigadores plantean en sus trabajos (Castellano, 2000; Costa, Garganta, Greco, Mesquita y Maia, 2001; Fernández-Navarro y col., 2016). En la figura 4 proponemos una adaptación de Castellano 2000 con cuatro divisiones horizontales y tres verticales (no obstante, estos espacios pueden ser adaptados a las necesidades concretas de cada equipo).

Protagonistas: determinados y clasificados por su influencia en la acción, de tal forma que los principales sería/ $\mathrm{n}$ aquel/aquellos que tienen una influencia directa con la acción o secundarios, si tienen una influencia indirecta con la acción.

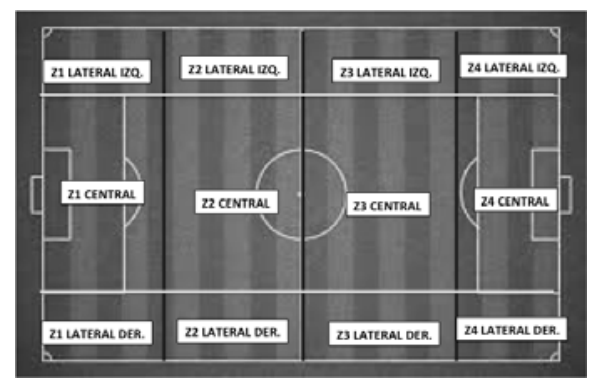

Figura 4. División del campo en zonas (adaptado de Castellano, 2000).

\section{$\varnothing$ Funciones específicas por puestos:}

Las funciones específicas por puesto son una parte de la guía que facilita la descripción y concreción de comportamientos en cada demarcación, desde el portero hasta los delanteros. Autores como Cano (2012) y Martín-Barrero (2016) hacen mencionado en sus trabajos sobre el MJ a este elemento. Estas funciones deben responder a preguntas como:

¿En qué fase/momento del juego?

¿Qué objetivos cumplen y cómo (a través de qué medios técnico-tácticos)?

¿Con quiénes interaccionan?

$\varnothing$ Patrones de juego a adoptar ante estrategias del rival:

Tal y como se mencionaba en el segundo punto de este trabajo, la flexibilidad es una característica importante que debe tener un $\mathrm{MJ}$. El rival puede plantear una estrategia que dificulte el desarrollo de los patrones de juego estableci- dos, es por ello que consideramos importante la introducción de este elemento como parte de la hoja de ruta que complete la guía propuesta. Este elemento puede ser definido como la modificación de los comportamientos principales según circunstancias adversas del juego. En esta línea, Martín-Barrero (2016) nos indica que esta adaptación de patrones debe ser considerada tanto para posibles estrategias ofensivas como defensivas del rival.

\section{$\varnothing \mathrm{ABP}:$}

Siguiendo a Castellano (2009) podemos definir las ABP como el conjunto de acciones que reanudan el juego después de una interrupción reglamentaria. FernándezHermógenes et al (2017) indican que las ABP son cada vez más frecuentes como elementos desequilibradores en el juego, de tal forma que las ABP ofensivas son cada vez más decisivas y pueden determinar el resultado de un partido entre equipos del mismo nivel (Castelo, 1999; Mombaerts, 2000). Debido a estas reflexiones, consideramos interesante que las $\mathrm{ABP}$ se tengan en cuenta como uno de elemento dentro del diseño de la guía sobre el MJ. La propuesta sobre las cuestiones que deben ser respondidas para la aplicación práctica de las ABP pueden ser:

- Tipo de ABP: ofensiva o defensiva.

- Procedimiento de actuación: comportamientos fundamentales a llevar a aplicar.

- Protagonistas: ejecutores, rematadores, defensores etc.

\section{Aplicación práctica de la hoja de ruta para la elabora- ción de un modelo de juego.}

A continuación y a partir de los diferentes elementos que conforman la hoja de ruta establecida se desarrollará una propuesta práctica de la misma. Esta propuesta práctica puede ser optimizada y adaptada según la necesidad del lector. Nuestro objetivo no es establecer un modelo cerrado, todo lo contrario, es ilustrar el sendero que ayude a los lectores a desarrollar su modelo propio a través de la reflexión y el análisis independientemente del momento y la circunstancia del equipo.

\section{Ø Plantilla (jugadores):}

\begin{tabular}{|c|c|c|}
\hline $\begin{array}{l}\text { Características } \\
\text { generales }\end{array}$ & Puntos fuertes & Puntos débiles \\
\hline $\begin{array}{l}\text { Portero. } \\
\text { Diestro. } \\
5 \text { años en el club. } \\
\text { Más de } 100 \text { partidos } \\
\text { en la Premier League. } \\
\text { Internacional con } \\
\text { Dinamarca en más de } \\
20 \text { ocasiones. }\end{array}$ & $\begin{array}{l}\text { Buena capacidad de salto, ágil y } 1.89 \text { m } \\
\text { Buen juego con los pies } \\
\text { Saque de puerta largo, lo que le hace } \\
\text { peligroso ante posibilidad de contraataque. } \\
\text { Buena salida en juego aéreo (valiente). } \\
\text { Buena capacidad bajo palos. } \\
\text { Tiende a tener buenos registros parando } \\
\text { penaltis. }\end{array}$ & $\begin{array}{l}\text { 1X1 y achicar espacios } \\
\text { ante situaciones de } \\
\text { rechaces. } \\
\text { Juego ofensivo cuando } \\
\text { tiene que ejecutarlo con } \\
\text { la pierna izquierda. }\end{array}$ \\
\hline
\end{tabular}

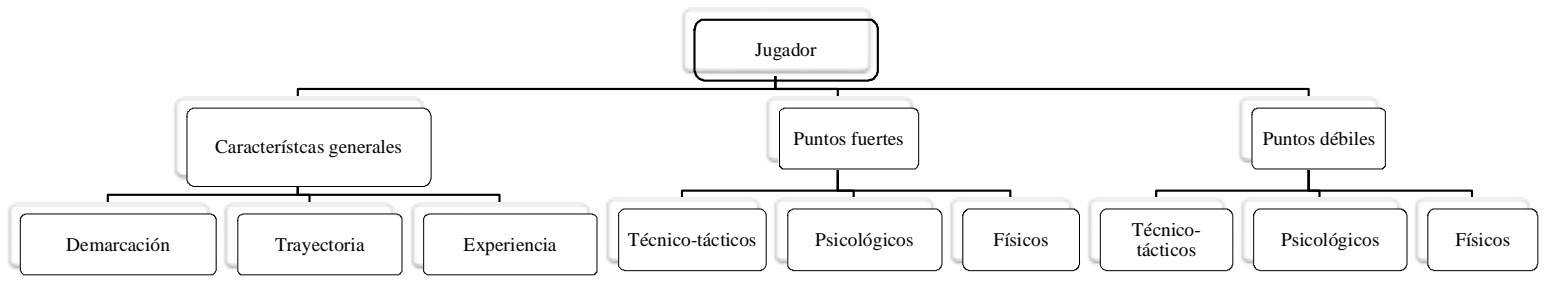

Figura 5. Características de los jugadores a definir para el diseño y análisis en el MJ y ejemplo práctico (Martín-Barrero, 2016). 
Ø Relación del ciclo del juego y objetivos secuenciales:

En el siguiente esquema se puede observar cómo se organizan por fases y momentos del juego los diferentes objetivos secuenciales, de tal forma que sirva de visualización y configuración previa a los patrones de juego.

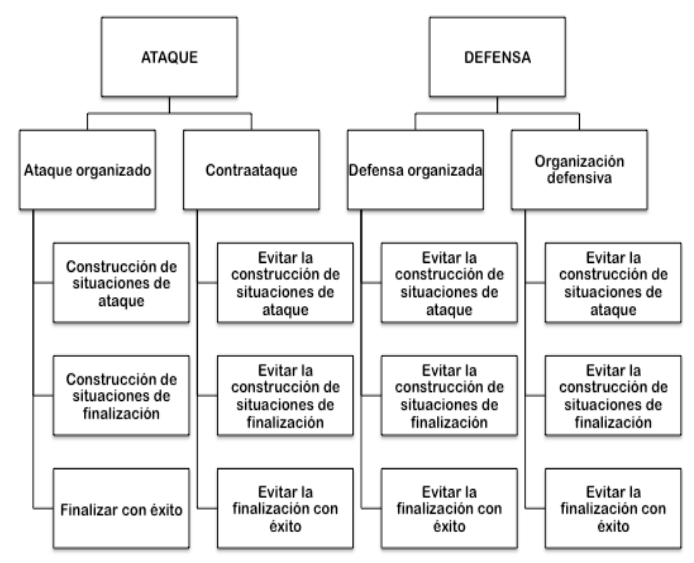

Figura 6. Relación ciclo del juego y objetivos secuenciales del juego.

\section{$\varnothing$ Patrones de juego:}

La definición y descripción de los patrones es uno de los matices más relevantes para el desarrollo del diseño y análisis del MJ. El aspecto más importantes es definir el conjunto de principios que defina comportamientos y proporcione organización al equipo (Mourinho citado en Gaiteiro, 2006). La claridad del mensaje ocupa también un papel relevante, siguiendo a Tamarit (2010) cuanto más elaborado está un MJ y mejor expuesto esté a los jugadores, más claridad tendrán los mismos sobre los diferentes comportamientos que tienen que adoptar. Por lo tanto, podríamos considerar de gran relevancia la organización de estos patrones y la coherencia de contenido de los mismos. A continuación desarrollamos una propuesta práctica que explique mediante esquemas posibles itinerarios. La primera de ellas es para el momento de ataque organizado y sus respectivos objetivos secuenciales.

Los patrones de juego para el ataque organizado tratarían de perseguir como mínimo los siguientes objetivos específicos, que a su vez deben permitir desarrollar los comportamientos:

o ATAQUEORGANIZADO:

o Objetivos en relación a la ocupación del espacio: ¿qué objetivo/s persigo con la ocupación de espacios?

o Objetivos en relación a como se pretende genera superioridad numérica: ¿qué objetivo/s persigo con la movilidad y la provocación de superioridades numéricas?

o Objetivos en relación al desequilibrio: ¿qué objetivo/ s persigo cuando busco desequilibrio o penetro?

Con respecto a los procedimientos de actuación, los cuales serán los componentes que den contenido a los objetivos específicos y tomarán como punto de partida los diferentes medios técnico-tácticos y comportamientos actitudinales.

\section{o ATAQUE ORGANIZADO (Figura 7):}

o Procedimientos de actuación para los objetivos de ocupación de espacios: ¿cómo ocupo los diferentes espacios? En estos procedimientos tendremos en cuenta medios técnico-tácticos como la amplitud y profundidad (Figura 8), posicionamiento y ocupación de zonas de remate.

o Procedimientos de actuación para los objetivos de generar superioridades numéricas: ¿dónde y cómo genero las diferentes superioridades numéricas? En estos procedimientos tendremos en cuanta medios técnico-tácticos como superioridad numérica sin balón, los desmarques, llegadas de segunda línea y los cambios de posición y rol.

o Procedimientos de actuación para los objetivos de desequilibrio: ¿cómo penetro o rompo las diferentes líneas defensivas del rival? Para estos tendremos en cuanta las circulaciones de balón y la generación de superioridades numéricas con balón, los centros y situaciones de tiro y remate.

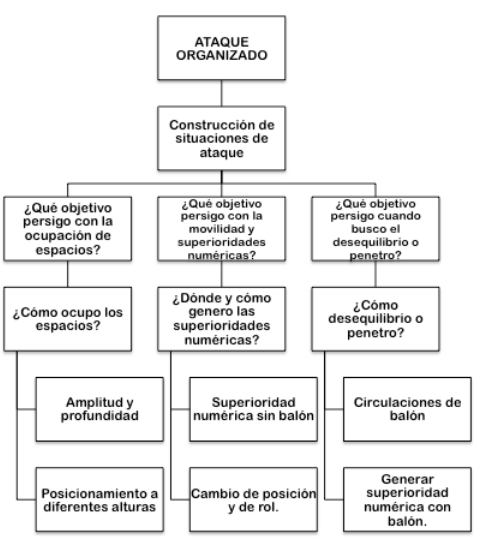

Figura 7. Hoja de ruta para organizar el contenido correspondiente a los patrones de juego en el momento de ataque organizado y persiguiendo el objetivo de construir situaciones de ataque.

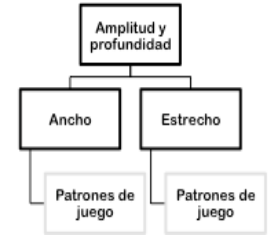

¿Como defino procedimientos relacionados con la amplitud y profundidad?

$\checkmark$ Distancias de relacionas largas o cortas y lo que se pretende generar con esas distancias de relación (separar al rival lo máximo posible o estar juntos para favorecer el juego directo o contraataque). $\checkmark$ Si hay intención o no de fijar alguna línea defensiva rival y como lo pretendo hacer.

Figura 8. Plan esquemático sobre cómo describir los diferentes comportamientos y procedimientos de actuación en relación a los medios técnico-tácticos de amplitud y profundidad.

Para los momentos de contraataque, los objetivos se enfocarían de manera diferente:

o CONTRAATAQUE:

o Objetivos relacionados con las primeras conexiones: ¿qué objetivo/s busco cuando el equipo recupera el balón y tiene que establecer las primeras conexiones en relación al balón?

o Objetivos en relación al desequilibrio/continuidad: una vez que consigo establecer las primeras conexiones, ¿̇mis objetivos están orientados a darle continuidad al ataque o desequilibrar?

En relación a los procedimientos de actuación (Figura9):

\section{o CONTRAATAQUE}

o Procedimientos de actuación en relación a las primeras conexiones: ¿cuáles y como son las primeras conexiones y a que zonas? y ¿cómo se ocupan los espacios para establecer esas primeras conexiones? Aquí entran medios técnicotácticos como el pase, amplitud y profundidad.

o Procedimientos de actuación en relación al desequilibrio o continuidad: ¿cómo es la circulación del balón y como y donde se generan las superioridades numéricas? Incluso si es en situación de finalización contemplar la llegada de 
jugadores de segunda línea. En este apartado habría que tener en cuenta medios técnico-tácticos como la conducción, pases o tiro (finalización).

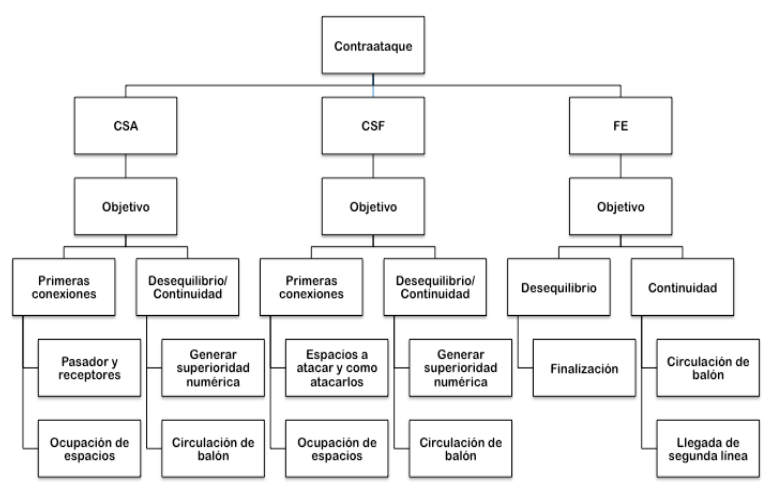

Figura 9. Hoja de ruta para organizar el contenido correspondiente a los patrones de juego en el momento de contraataque para cada uno de los OSJ.

A nivel defensivo la relación será acorde a los momentos defensivos del juego, de tal forma que los objetivos cambiarían (Figura 10):

o DEFENSA ORGANIZADA:

o Objetivos en relación a la ocupación del espacio defensivo: ¿qué objetivos persigo con la ocupación de espacios?

o Objetivos en relación al robo del balón: ¿qué objetivos persigo cuando intento robar o recuperar la posesión del balón?

o Objetivos en relación a la actuación defensiva cuando el equipo es desequilibrado: ¿objetivos cuando me desequilibran?

En relación a los procedimientos y comportamientos defensivos, estos también varían:

o DEFENSAORGANIZADA:

o Procedimientos de actuación para los objetivos de ocupación de espacios defensivos: ¿cómo y qué espacios ocupo? Tomarán relevancia los medios técnico-tácticos como el bloque defensivo (la altura del mismo) y los marcajes.

o Procedimientos de actuación para los objetivos robo de balón: ¿dónde y cómo genero o provoco la recuperación del balón? Aquí estarían presente los medios técnico-tácticos de presión y presencia intensiva.

o Procedimientos de actuación para los objetivos de desequilibrio: ¿cómo actúo cuando me desequilibran? Rela-

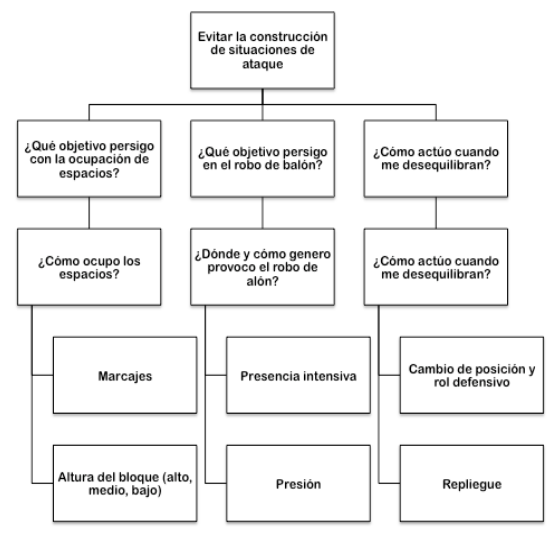

Figura 10. Hoja de ruta para organizar el contenido correspondiente a los patrones de juego en el momento de defensa organizada y persiguiendo el objetivo de evitar la construcción de situaciones de ataque. cionado con estos procedimientos estarían los medios técnico-tácticos de cambio de posición y rol defensivo y repliegue.

En los siguientes esquemas se muestran dos ejemplos de la idea expuesta anteriormente, pretendiendo estructurarlo como una hoja de ruta la cual que facilite el plan organizativo.

A su vez los procedimientos de actuación pueden ser representados de una manera similar, de tal forma que pueda servir de plan para describir los diferentes comportamientos. En el siguiente esquema (Figura 11) se expone un ejemplo de lo que se pretende.
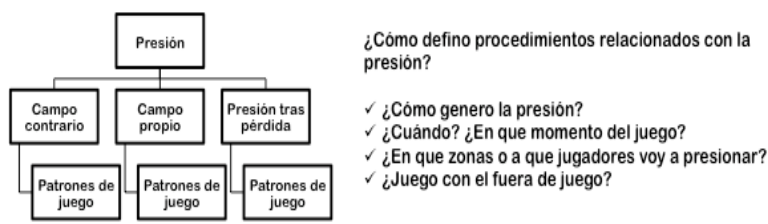

Figura 11. Plan esquemático sobre cómo describir los diferentes comportamientos y procedimientos de actuación en relación al medio técnico-táctico de presión.

En relación a la organización defensiva la propuesta sería la que se expone a continuación (Figura 12):

o ORGANIZACIÓNDEFENSIVA:

o Objetivos en relación a la acción defensiva cuando el equipo pierde la posesión del balón: ¿qué objetivos persigo cuando pierdo la posesión del balón?

o Objetivos en relación a la reorganización: ¿qué objetivos persigo cuando el equipo es superado y necesita reorganizarse?

Con respecto a los procedimientos de actuación:

o ORGANIZACIÓNDEFENSIVA

o Procedimientos de actuación en relación a la acción defensiva: ¿cómo robo y en qué zonas?

o Procedimientos de actuación en relación a la reorganización: ¿cómo es el repliegue y en qué zonas?

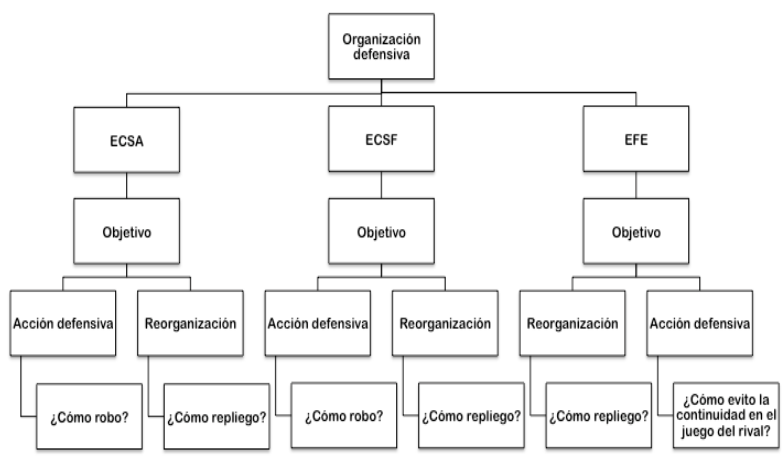

Figura 12. Hoja de ruta para organizar el contenido correspondiente a los patrones de juego en el momento de organización defensiva para cada uno de los OSJ.

A continuación se expone un ejemplo de cómo podría llevarse a la práctica una de las hojas de ruta expuestas anteriormente, concretamente para la construcción de situaciones de finalización en el momento de ataque organizado (Tabla 3).

$\varnothing$ Funciones específicas por puesto:

Con respecto a la hoja de ruta para definir las funciones específicas por puestos, estaría compuesta por cada uno de los momentos del juego para posteriormente definir numeradamente cada una de las funciones más importantes 
Tabla 3.

Ejemplo propuesto como aplicación práctica de la hoja de ruta para la construcción de situaciones de finalización en el ataque organizado.

\begin{tabular}{|c|c|c|}
\hline \multicolumn{3}{|c|}{ CONSTRUIR SITUACIONES DE FINALIZACIÓN: Conseguir situaciones de desequilibrio. } \\
\hline $\begin{array}{c}\text { OBJETIVOS } \\
\text { ESPECÍFICOS }\end{array}$ & PROCEDIMIENTOS DE ACTUACIÓN & $\begin{array}{c}\text { JUGADORES } \\
\text { PROTAGONISTAS }\end{array}$ \\
\hline $\begin{array}{l}\text { Disponer posicionalmente } \\
\text { la ocupación del espacio } \\
\text { con el fin de posibilitar las } \\
\text { diferentes relaciones con } \\
\text { balón y desajustar la } \\
\text { organización inicial del } \\
\text { rival }\end{array}$ & $\begin{array}{l}\text { *Posicionamiento de los jugadores en } \\
\text { amplitud para ocupar espacios laterales. } \\
\text { *Posicionamiento de los jugadores en } \\
\text { profundidad para provocar la fijación de } \\
\text { línea defensiva rival y poder facilitar la } \\
\text { llegada de jugadores de segunda línea. } \\
\text { *Posicionamiento en pocas alturas } \\
\text { permitiendo al equipo ser más transversales. } \\
\text { *Distancias de relación que permitan } \\
\text { disgregar los espacios entre líneas } \\
\text { defensivas en los pasillos laterales. } \\
\text { *Poca acumulación de jugadores por delante } \\
\text { del balón. }\end{array}$ & TODOS \\
\hline $\begin{array}{l}\text { Llegar a zonas cercanas al } \\
\text { área a través de generación } \\
\text { de espacios en pasillos } \\
\text { laterales }\end{array}$ & $\begin{array}{l}\text { *Búsqueda de circulación directa de un lado } \\
\text { débil a lado fuerte. } \\
\text { *Estabilidad en el ritmo de circulación de } \\
\text { balón. } \\
\text { *Insistencia en acciones de centro al área } \\
\text { tras posible despeje de la defensa contraria. }\end{array}$ & $\begin{array}{l}\text { CENTRALES, } \\
\text { MEDIOCENTROS Y } \\
\text { LATERALES }\end{array}$ \\
\hline $\begin{array}{l}\text { Posibilitar opciones de } \\
\text { progresión a jugadores o } \\
\text { espacios cercanos a } \\
\text { situaciones de finalización } \\
\text { generando desequilibrios } \\
\text { que obliguen } \\
\text { constantemente a la } \\
\text { defensa rival a retroceder o } \\
\text { recomponer sus líneas. } \\
\end{array}$ & $\begin{array}{l}\text { *Prioridad de penetración por pasillo lateral } \\
\text { con el fin de generar centros al área rival. } \\
\text { *Generar superioridad numérica en pasillos } \\
\text { laterales para poder progresar. } \\
\text { *Verticalidad ante situaciones de } 1 \text { x1 de los } \\
\text { jugadores con más habilidad. } \\
\text { *Predisposición constante a buscar y recibir } \\
\text { en espacios de penetración por detrás de la } \\
\text { última línea defensiva rival. }\end{array}$ & $\begin{array}{l}\text { MEDIOCENTROS, } \\
\text { INTERIORES, } \\
\text { SEGUNDO PUNTA } \\
\text { Y PUNTA }\end{array}$ \\
\hline
\end{tabular}

teniendo en cuenta los objetivos específicos, comportamientos o procedimientos, interacciones, actitudes o matices particulares de la demarcación.
Posición
o Funciones para el ataque organizado
$\S$ Funciones 1,2,3 ...
o Funciones para la organización defensiva
$\S$ Funciones 1,2,3 ...
o Funciones para la defensa organizada
$\S$ Funciones $1,2,3 \ldots$
o Funciones para el contraataque
$\S$ Funciones $1,2,3 \ldots$

Tabla 4.

Ejemplo propuesto para la demarcación de defensa central en la organización de los contenidos para establecer las funciones específicas por puesto.

\begin{tabular}{|c|c|c|c|}
\hline \multicolumn{4}{|c|}{ CENTRALES } \\
\hline $\begin{array}{c}\text { ATAQUE } \\
\text { ORGANIZADO }\end{array}$ & $\begin{array}{l}\text { ORGANIZACIÓN } \\
\text { DEFENSIVA }\end{array}$ & $\begin{array}{c}\text { DEFENSA } \\
\text { ORGANIZADA }\end{array}$ & CONTRAATAQUE \\
\hline $\begin{array}{l}\text { Establecer un } \\
\text { posicionamiento que } \\
\text { permita orientar la } \\
\text { trayectoria del balón } \\
\text { hacia los pasillos } \\
\text { laterales. } \\
\text { Intentar llevar el balón } \\
\text { a zonas lo más } \\
\text { adelantadas posibles. } \\
\text { Orientar las } \\
\text { circulaciones de balón } \\
\text { hacia los pasillos } \\
\text { laterales. } \\
\text { Evitar riesgos o } \\
\text { acciones de juego } \\
\text { interior que puedan } \\
\text { provocar pérdidas en } \\
\text { la posesión del balón. } \\
\text { Posibilitar la } \\
\text { continuación del juego } \\
\text { ante retroceso de la } \\
\text { circulación del balón. }\end{array}$ & $\begin{array}{l}\text { Vigilar y controlar a } \\
\text { los jugadores más } \\
\text { avanzados del equipo } \\
\text { rival. } \\
\text { Evitar o incomodar la } \\
\text { recepción de } \\
\text { jugadores más } \\
\text { avanzados del equipo } \\
\text { rival. } \\
\text { Retroceder para ganar } \\
\text { tiempo en caso de } \\
\text { recepción de jugador } \\
\text { del equipo rival. } \\
\text { Evitar la penetración } \\
\text { por pasillo central } \\
\text { ante situaciones } \\
\text { avanzadas de los } \\
\text { atacantes rivales. } \\
\text { Retrasar lo máximo } \\
\text { posible cualquier } \\
\text { situación de posible } \\
\text { finalización. }\end{array}$ & $\begin{array}{l}\text { 1.Evitar distancias de } \\
\text { relación defensiva que } \\
\text { provoquen espacios de } \\
\text { penetración. } \\
\text { 2. Permanecer como un } \\
\text { bloque compacto con } \\
\text { los demás integrantes de } \\
\text { su propia línea } \\
\text { defensiva y las líneas } \\
\text { consecuentes. } \\
\text { 3. Achicar espacios ante } \\
\text { balón que se introduzca } \\
\text { entre líneas. } \\
\text { 4. Acosar al jugador } \\
\text { receptor de balón en } \\
\text { espacios entre líneas. } \\
\text { 5. No conceder espacios } \\
\text { de penetración } \\
\text { interlineas. } \\
\text { 6. No abandonar el } \\
\text { pasillo central a menos } \\
\text { que requiera la ayuda } \\
\text { del lateral. } \\
\text { 7. Evitar recepciones } \\
\text { del rival dentro del área, } \\
\text { siendo los principales } \\
\text { protectores de la } \\
\text { portería. }\end{array}$ & $\begin{array}{l}\text { Conectar con } \\
\text { jugadores más } \\
\text { avanzados que } \\
\text { permitan la } \\
\text { construcción del } \\
\text { contraataque. } \\
\text { Evitar avanzar hasta } \\
\text { tener asegurada la } \\
\text { posesión del balón } \\
\text { permitiendo dar } \\
\text { continuidad en el } \\
\text { juego. }\end{array}$ \\
\hline
\end{tabular}

Ø Patrones de juego a adoptar ante estrategias del rival: Siguiendo la misma idea llevada a cabo en las anteriores propuestas, a continuación se propone una hoja de ruta que ayude a definir el contenido a aplicar en cada uno de los matices de este elemento del MJ. En la Figura 13 se refleja el ejemplo práctico aplicado por Martín-Barrero (2016).

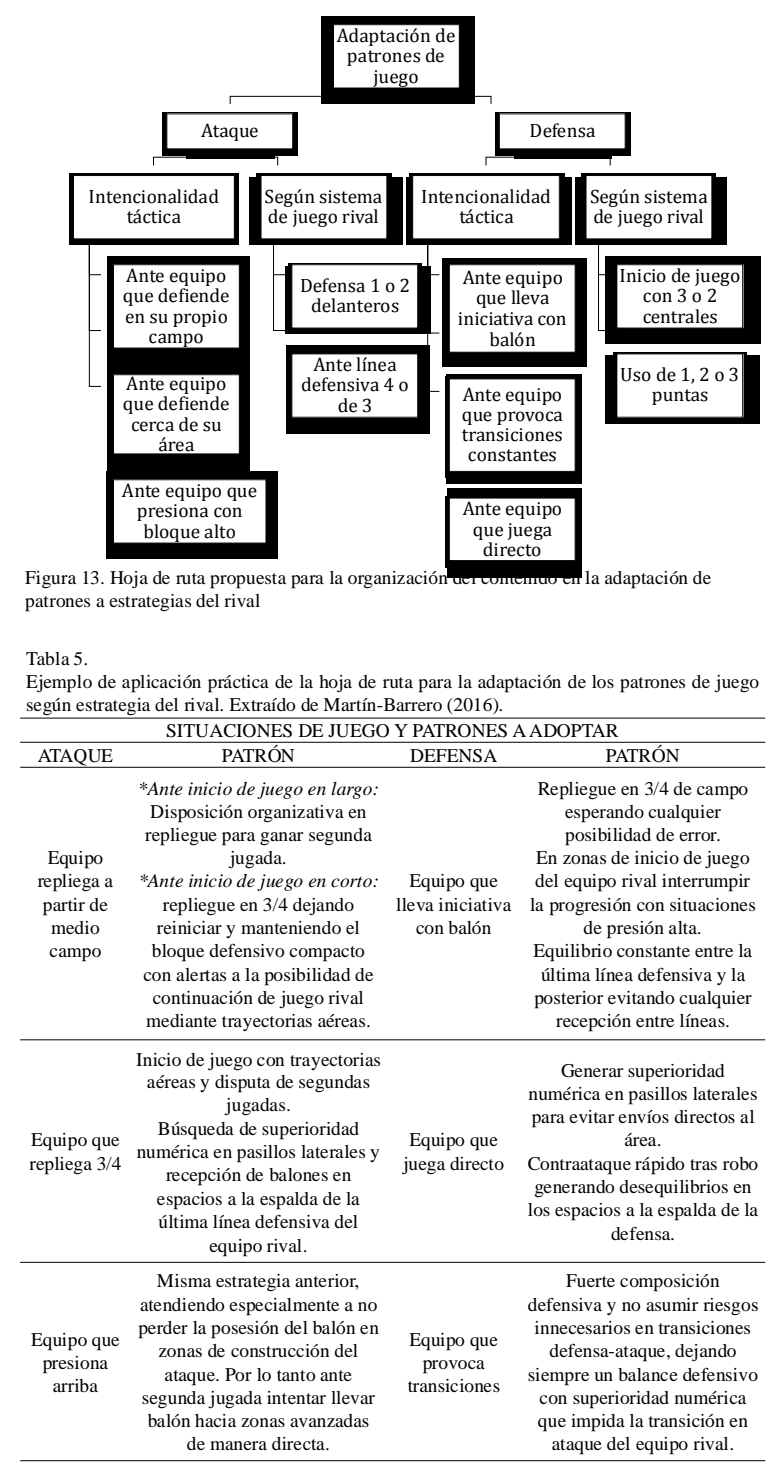

\section{Conclusión}

La competición en el fútbol actual exige al entrenador tener organizado y optimizado todos los recursos de su equipo. Dentro de los diferentes recursos que pueda tener el entrenador y el cuerpo técnico dentro de un club, podemos determinar que el $\mathrm{MJ}$ es una herramienta muy útil y de bajo coste que permite establecer los pilares fundamentales sobre los que se sostendrán el juego del equipo. Además tener desarrollada y bien fundamentada dicha propuesta podría ayudar a identificar el «que», «como» y «donde» puede mejorar el rendimiento el equipo. Estos parámetros pueden servir en todo momento de guía y de hoja de ruta, teniendo en cuenta que una de las características más importantes que debe de tener cualquier MJ es la flexibilidad para ser modificado siempre que las circunstancias lo requieran.

Por lo tanto, el MJ debe servirnos como guía que identifique y organice cada uno de los pilares o elementos (desde los jugadores hasta las ABP) que conforman un equipo de fútbol, a la misma vez, que se extiende al contexto de entrenamiento, ya que este nos sirve para elaborar y definir los prin- 
cipales contenidos que se establezcan en las diferentes sesiones durante el periodo de entrenamiento.

Nos gustaría concluir este trabajo resaltando que la propuesta presentada con sus correspondientes ejemplos y preguntas planteadas pueden ser ampliadas, adaptadas y/o mejoradas siempre que se considere oportuno para el entrenamiento y desarrollo del MJ.

\section{Bibliografía}

Amiero, N. (2005). Defensa en zona en el fútbol. Barcelona: MCSport. Balagué, N. \& Torrents, C. (2011). Complejidad y deporte (Rendimiento deportivo). Barcelona: Inde.

Bangsbo, J. \& Peitersen, B. (2000). Soccer systems and strategies. Champaign, IL: Human Kinetics.

Bate, R. (1988). Football chance: Tactics and strategy. In T. Reilly, A. Lees, K. Davids, \& W. J. Murphy (Eds.), Science and football (pp. 293-301). London: E \& FN Spon.

Cano, O. (2009). El modelo de juego del FC Barcelona. Barcelona: MCSport.

Cano, O. (2012). El juego de posición del FC Barcelona. Barcelona: MCSport.

Carvalhal, C. (2001). No treino de futebol de rendimento superior: a recuperação é muitíssimo mais que recuperar. Braga.

Castellano, J. (2000). Observación y análisis de la acción de juego en el fútbol (Tesis doctoral inédita). Universidad del País Vasco.

Castellano, J. (2008). Análisis de las posesiones de balón en fútbol: Frecuencia, duración y transición. European Journal of Human Movemeny, 21(0), 189-207.

Castellano, J. (2009). Conocer el pasado del fútbol para cambiar su futuro. Acción motriz. Tu revista científica digital, 2, 37-50.

Castellano, J., Perea, A. \& Álvarez, D. (2009). Transiciones en la posesión del balón en fútbol: de lo posible a lo probable. Apunts. Educación Física y Deportes, 95, 75-81.

Castelo, J. (1994). Modelo técnico-táctico do jogo. Lisboa: Ediçoes FMH.

Castelo, J. (1999). Fútbol: estructura y dinámica del juego. Barcelona: Paidotribo.

Costa, I., Garganta, J., Greco, P., Mesquita, I., \& Maia, J. (2011) Sistema de avaliação táctica no Futebol (FUT-SAT): Desenvolvimento e validação preliminar. Revista Motricidade, 7 (1).

Delgado, J., \& Méndez-Villanueva, A. (2012). Tactical Periodization: Mourinho's best-kept secret? Soccer Journal, 57 (3), 29.

Drust, B., Atkinson, G., y Reilly, T. (2007). Future perspectives in the evaluation of the physiological demands of soccer. Sports Medicine, 37(9), 783-805.

Fernandes, V. (2003): Implementação do Modelo de Jogo: da razão à adaptabilidade com emoção. Facultad de Deporte de la Universidad de Porto, Porto.

Fernández-Hermógenes, D., Camerino, O., \& García, A. (2017). Acciones ofensivas a balón parado en el fútbol. Apunts. Educación Física y Deportes, 123, 78-94.

Fernández-Navarro, J., Fradua, L., Zubillaga, A., Ford, P. \& McRobert, A. (2016). Attacking and defensive styles of play in soccer: analysis of Spanish and English elite teams. Journal of Sports Sciences, 34(24), 2195-2204.

Frade, V. (1985). Alta competiçao no futebol-que exigencias do tipo metodológico? Facultad de Deporte de la Universidad de Porto, Porto

Frade, V. (2004). Entrevista en P. Leal (2004). Diferentes entendimentos, diferentes orientações metodológicas. Facultad de Deporte de la Universidad de Porto, Porto.

Freitas S. (2004). A especificidade que está na concentração táctica que está na ESPECIFICIDADE... no que deve ser uma operacionalização da Periodização Táctica [monografía]. Universidad de Porto, Porto.

Gaiteiro, B. (2006). A Ciência oculta do sucesso: Mourinho aos olhos da ciencia. Facultad de Deporte de la Universidad de Porto, Por- to.

Garganta, J. (2016). La humanización del entrenamiento y la competición será la gran revolución del fútbol. Fútbol holístico. Recuperado de https://holistico.wordpress.com/2016/04/06/julio-garganta-la-humanizacion-del-entrenamiento-y-la-competicionsera-la-gran-revolucion-del-futbol/ el 15/12/2018.

Guindos, D. (2015). Construcción metodológica del modelo de juego. Madrid: Futboldlibro.

Garganta, J., Maia, J., \& Basto, F. (1997). Analysis of goal-scoring patterns in European top level soccer teams. En Bangsbo, J., Reilly, T., \& Williams, A.M. (1997). Science and football III (pp. 246-250). London: E \& FN Spon.

Garganta, J., \& Pinto, J. (1998). O Ensino do Futebol. En Graça, A., \& Oliveira, J. (Eds.), O ensino dos jogos deportivos, $3^{\mathrm{a}} \mathrm{Ed}$. (pp. 95 - 135). Porto: Centro de Estudos dos Jogos Desportivos. Facultad de Deporte de la Universidad de Porto, Porto.Gurutz, J. (2012). Los sistemas de juego. Abfutbol, 60,15-31.

Hewitt, A., Greenham, G., \& Norton, K. (2017). Game style in soccer: what is it and can we quantify it? International Journal of Performance Analysis in Sport, 16(1), 355-372.

Hughes, M., \& Franks, I. (2005). Analysis of passing sequences, shots and goals in soccer. Journal of Sports Sciences, 23(5), 509-514.

Lopez, J. (2004). Modelos tácticos y sistemas de juego: elaboración y entrenamiento integrado. Sevilla: Wanceulen.

Marcos, J.M. (2011). La Metodología de la estrategia operativa como Plan Estratégico para cada partido. Fútbol-táctico, 49.

Martín-Barrero, A. (2016). El modelo de juego del Leicester City. De la concepción teórica a la aplicación práctica. Abfutbol, 82, 1354.

Martín-Barrero, A. (2018). ¿Cómo construir el camino desde el fútbol de la calle al fútbol profesional? Madrid: Abfutbol.

Mercé, J. (2004). Fútbol: el sistema 1.4.4.2.: fundamentos y enseñanza. Sevilla: Wanceulen.

Mombaerts, E. (2000). Fútbol. Del análisis del juego a la formación del jugador. Barcelona: INDE.

Mourinho, J. (2014). The Daily Telegraph [entrevista]. Recuperado de https://www.telegraph.co.uk/

Oliveira, J.G. (2004). Conhecimento Específico em Futebol. Contributos para a definição de uma matriz dinâmica do proceso ensinoaprendizagem/ treino do jogo. Facultad de Deporte de la Universidad de Porto, Porto.

Pascual, N. (2012). Análisis comparativo de los sistemas de juego en el fútbol siete alevín (Tesis doctoral inédita). Universidad de Alicante, Alicante.

Reilly, T. (2005). An ergonomic model of the soccer training process. Journal of Sport Sciences, 23(6), 561-572.

Ruiz-Ruiz, C., Fradua, L., Fernández-García, Á., \& Zubillaga, A. (2013). Analysis of entries into the penalty area as a performance indicator in soccer. European Journal of Sport Science, 13(3), 241-248.

Serra, J., García, L., \& Sánchez-Mora, D. (2011). El juego modificado, recurso metodológico en el fútbol de iniciación. Retos: Nuevas tendencias en educación física, deporte y recreación, 20, 37-42.

Silva, P; Oliveira, P. \& Kautzner, N. (2009). Treinar futebol respeitando a essência do jogo: o exemplo do salto como ação tática e não somente técnico-física. Faculdade de Educação Física da Universidad Estatal de Campinas, 7(2), 38-63.

Tamarit, X. (2010). ¿Qué es la periodización táctica? Barcelona: McSport.

Tenga, A., \&y Larsen, O. (2003). Testing the validity of match analysis to describe playing styles in football. International Journal of Performance Analysis in Sport, 3(2), 90-102.

Teodorescu, L. (1984). Problemas de teoria e metodogia nos jogos desportivos. Lisboa: Livros Horizonte.

Vales-Vázquez, A. (1998). Propuesta de indicadores tácticos para la optimización de los sistemas de juego en el fútbol. Tesis doctoral (inédita). Universidad de Coruña. A Coruña.

Wright, C., Atkins, S., Polman, R., Jones, B., \& Sargeson, L. (2011). Factors associated with goals and goal scoring opportunities in profesional Soccer. International Journal of Performance Analysis in Sport, 11(3), 438-449. 